\title{
Novel Drug Delivery Strategies in Neuro-Oncology
}

\author{
Dani S. Bidros and Michael A. Vogelbaum \\ Department of Neurological Surgery, Brain Tumor and NeuroOncology Center, Neurological Institute, \\ Cleveland Clinic, Cleveland, Ohio 44195
}

\begin{abstract}
Summary: Treatment of malignant gliomas represents one of the most formidable challenges in oncology. Despite treatment with surgery, radiation therapy, and chemotherapy, the prognosis remains poor, particularly for glioblastoma, which has a median survival of 12 to 15 months. An important impediment to finding effective treatments for malignant gliomas is the presence of the blood brain barrier, which serves to prevent delivery of potentially active therapeutic compounds. Multiple efforts are focused on developing strategies to effectively deliver active drugs to brain
\end{abstract}

tumor cells. Blood brain barrier disruption and convectionenhanced delivery have emerged as leading investigational delivery techniques for the treatment of malignant brain tumors. Clinical trials using these methods have been completed, with mixed results, and several more are being initiated. In this review, we describe the clinically available methods used to circumvent the blood brain barrier and summarize the results to date of ongoing and completed clinical trials. Key Words: Convection-enhanced delivery, blood brain barrier disruption, brain neoplasm, drug delivery system.

\section{INTRODUCTION}

Malignant glioma is the most common type of primary brain tumors in adults. According to the Central Brain Tumor Registry of the United States, it is estimated that there are approximately 20,000 new cases each year. ${ }^{1}$ Treatment of malignant gliomas represents one of the most formidable challenges in oncology. Despite surgical and medical advancements, the 5-year survival rate for glioblastoma multiforme (GBM) in the United States has remained at a dismal $3.4 \%$ for the past 3 decades. $^{2}$ After recurrence, rapid tumor progression results in a median progression free survival and overall survival of 9 weeks and 25 weeks, respectively. ${ }^{3}$ The invasiveness of these tumors eliminates the possibility of curative surgical resection. Even at initial presentation, infiltration by tumor cells extends at least $2 \mathrm{~cm}$ away from the radiographic contrast-enhancing mass. ${ }^{4}$ An important impediment to the successful treatment of malignant gliomas is the presence of the blood brain barrier (BBB) and the partially functional blood tumor barrier, which serve to prevent effective delivery of active therapeutic compounds. There is vast interest in and consequently

Address correspondence and reprint requests to: Michael A. Vogelbaum, M.D., Ph.D., F.A.C.S., Brain Tumor and NeuroOncology Center, Neurological Institute, Cleveland Clinic, 9500 Euclid Ave, Cleveland, OH 44195. E-mail: vogelbm@ccf.org. multiple research efforts being focused on the design of new approaches that will improve drug delivery to brain tumor cells without producing dose-limiting systemic or neurological toxicity. This review will summarize some of the methods that have been developed for overcoming, or bypassing, the BBB with focus on direct-drug delivery and convection-enhanced delivery (CED).

\section{BARRIER DISRUPTION STRATEGIES FOR ENHANCING DRUG DELIVERY}

Although advances in neuropharmacology have led to the use of chemical modification techniques to make drugs more likely to reach the brain parenchyma in the setting of an intact $\mathrm{BBB}$, to date, the bulk of clinical investigation has focused on methods to either disrupt or circumvent the BBB. A variety of techniques that transiently disrupt the BBB have been investigated by creating a paracellular route of transport through the endothelium. These approaches have included opening tight junctions between endothelial cells and creating a transcellular pathway through the endothelium. Several endogenous compounds including neurotransmitters, hormones, and inflammatory mediators can readily open the tight junctions of the BBB. ${ }^{5}$ Disruption of the BBB has also been seen in physiological states, such as hypertension, hypoxia, or ischemia. Hypertonic substances including mannitol and biologically active agents, such as 
bradykinin and angiotensin peptides have also shown to disrupt the BBB.

\section{Osmotic BBB disruption}

Transient osmotic disruption of the BBB, and bloodCSF and blood-tumor barriers can be achieved throughout a vascular circulation by intra-arterial (IA) infusion of a hyperosmotic agent, which is usually mannitol. ${ }^{6} \mathrm{By}$ injecting hypertonic solution, rapid diffusion of fluid out of the cells occurs, causing shrinkage of endothelial cells and subsequent opening of the tight junctions of the BBB for several hours. ${ }^{7}$ This infusion of hypertonic solution is usually followed by administration of IA chemotherapy. Animal studies suggest that this method is able to increase concentrations of chemotherapeutic agents in the brain parenchyma up to 90 -fold. ${ }^{8}$ Methotrexate delivery to CNS is enhanced four- to seven-fold when administered IA after osmotic BBB disruption compared with IA administration without blood brain barrier disruption (BBBD). ${ }^{9}$ The ability of BBBD to improve therapeutic efficacy of chemotherapy has been variable in humans. In chemoresponsive tumors, BBBD chemotherapy compared favorably with conventional chemotherapy. ${ }^{10-12}$ In primary central nervous system lymphoma phase II studies, a significant difference was found (compared to historical controls) when comparing patients treated with chemotherapy, in the setting of BBBD, with or without prior whole-brain radiotherapy. ${ }^{13} \mathrm{~A}$ concern associated with the use of BBBD is the potential for neurotoxicity from the high concentrations of chemotherapy delivered to the normal brain. Chemotherapeutics, such as doxorubicin, cisplatin, and taxanes cause neurotoxicity with BBBD, even though they are systemically well tolerated. ${ }^{14}$ The efficacy of BBBD-mediated chemotherapy for malignant gliomas is still being explored.

\section{Biochemical BBBD}

This is a less invasive and possibly a more reliable technique for disruption of the BBB because it primarily affects the tumor vasculature. Mediators of inflammatory response, including leukotrienes, histamine and vasoactive peptides, have been found to cause transient vascular leakage and increased permeability of blood vessels. ${ }^{15}$ Bradykinin, a peripheral vasodilator, increases the tight junction permeability by activating B2 receptors of the endothelial cells. ${ }^{16}$ Previous studies have shown that the bradykinin agonist, RMP-7, can safely permeabilize the BBB. Animal models were used to compare the effects of RMP-7 within tumor, brain tissue proximal to tumor, and brain tissue distal from tumor. In one study, the effect of RMP-7 on enhancing BBB permeation was examined in combination with three compounds of different physical characteristics (i.e., carboplatin, dextran, and N,N'-bis(2-chloroethyl)-N-nitroso-urea [BCNU]). RMP-7 increased permeability of the vascular barriers to the hydrophilic compounds carboplatin and dextran. A greater impact of RMP-7 was observed in regions of the blood tumor barrier compared to nontumor BBB. RMP-7 had no obvious impact on permeabilizing the BBB for the lipophilic drug BCNU. In a multinational clinical trial, intra-arterial RMP-7 was evaluated in combination with the anticancer agent carboplatin for the treatment of malignant brain tumors. ${ }^{17}$ Due to high levels of toxicity, however, this clinical trial was discontinued.

\section{Ultrasound-mediated BBBD}

BBBD disruption over a large volume, as seen with osmotic and biochemical disruption, may prove a disadvantage for many applications, because some therapeutic agents may have undesired side effects as a result of their widespread distribution within the CNS. To prevent these side effects, a localized and reversible imageguided disruption of the BBB could provide anatomically or functionally targeted drug delivery while preserving an intact $\mathrm{BBB}$ to protect the nontargeted regions. A new approach to focal CNS delivery is BBB disruption by MRI-guided, focused ultrasound. ${ }^{18}$ Ultrasound-mediated BBB disruption has been shown to produce consistent vascular leak without tissue damage. This result was achieved by localizing cavitation-generated mechanical stresses to blood vessel walls by intravenous injection of preformed gas bubbles just before pulsed ultrasound treatment. ${ }^{19}$ The focal opening of the BBB was reversible and completely healed within 24 hours. A rat brain study showed that the locations of the brain that were exposed to ultrasound showed significantly higher concentrations of liposomal doxorubicin, and showed that clinically relevant levels were reached. ${ }^{20}$ Of note, the attenuation of the ultrasound by the cranium requires the creation of a bone window to deliver this treatment. However, recent studies have shown that focal, transcranial ultrasound treatment of brain tissue may be accomplished using a large surface area-phased array. ${ }^{21}$

\section{Methods for delivery of drugs directly to the brain parenchyma}

Conventional methods for the treatment of brain tumors usually involve delivery of drugs via the systemic circulation. High systemic drug levels are often required to achieve adequate drug concentrations at the site of the brain tumor, which usually requires increasing the dose, frequency, or duration of drug administration with consequent systemic toxicity. Strategies to enhance drug delivery to brain tumor cells by methods that do not rely on the circulatory system have been investigated. These physical-based techniques circumvent the BBB completely to improve brain drug delivery and hence obviate the need for drug manipulation by chemical-based techniques.

Interstitial drug delivery has been most appealing for the treatment of primary brain tumors, because it provides the most direct method of overcoming the barriers 
to tumor drug delivery. By administering therapeutic agents directly to the brain interstitium, and more specifically to tumor parenchyma, one can overcome the elevated interstitial pressure produced by brain tumors. Interstitial pressure gradients and drug concentration gradients favor drug redistribution from the center to the periphery of the tumor by a combination of convection and diffusion. Approaches to local drug delivery include the use of implantable controlled release polymer systems, various catheter devices for intracavitary drug delivery, and convection-enhanced delivery.

\section{Implanted polymers}

The use of implanted, biodegradable polymers in the treatment of brain tumors has been utilized in a number of clinical trials. The underlying concept is to provide continuous drug delivery to the tumor using a drugimpregnated wafer that has a controlled, sustained release rate. Brem et al. ${ }^{22}$ studied the treatment of gliomas using carmustine-loaded biodegradable polymers as a drug-delivery wafer. This matrix releases the drug by a combination of diffusion and hydrolytic polymer degradation. In their initial randomized, clinical trial, they were able to show an increase in mean survival time for patients with recurrent gliomas. This result led to the first Food and Drug Administration-approved polymer wafer, Gliadel (Eisai, Woodcliff Lake, NJ). A subsequent, randomized trial in patients with newly diagnosed malignant gliomas led to expanded indication from the Food and Drug Administration to include patients with either newly diagnosed or recurrent glioma who are undergoing a gross total tumor resection. ${ }^{23}$ Although these studies provided sufficient evidence of efficacy to gain Food and Drug Administration-approval, the magnitude of benefit was modest, short-lasting, and the risk of toxicity was not trivial. Additional preclinical trials have taken place utilizing polymers impregnated with chemotherapeutics such as paclitaxel, adriamycin, 5-fluorouracil, mitomycin, nimustin hydrochloride, and mitoxantrone. ${ }^{24-28}$ At the time this article was written, these combinations had not been commercially developed. The disadvantages of these local delivery strategies include the increased risk of local neurotoxicity with higher polymer/drug concentrations, poor wound healing, and limited drug penetration beyond the resection cavity, which fails to provide therapeutic drug concentrations to tumor cells that are centimeters away.

\section{Intra-cavitary delivery systems}

The use of an intraventricular or intracavitary system, such as an Ommaya reservoir, allows for delivery of intermittent bolus injections of anticancer drugs directly to a tumor cyst or cavity. Other systems are capable of delivering drugs at a constant infusion over a prolonged period of time at a desired rate to the site of the intracranial tumor by the outlet catheter. Drugs such as ni- trosourea and methotrexate have been used in various clinical trials with good results. Infection, catheter obstruction, and inadequate drug distribution limits the success of these delivery methods.

\section{Convection-enhanced delivery}

A more recently described approach for achieving both local and regional drug delivery was proposed by Bobo et al. ${ }^{29}$ in 1994. Convection-enhanced delivery (CED) of substances within the human brain is becoming a more frequent experimental treatment option in the management of brain tumors, and more recently in phase 1 trials for gene therapy in Parkinson's disease. ${ }^{30}$ This approach involves the continuous injection under positive pressure of a solute containing a therapeutic agent. CED relies on pressure-driven bulk flow of infusate as a means to deliver therapeutic agents to the CNS. The bulk flow mechanism is created by a small pressure gradient from a pump that pushes solute through a catheter targeted within the CNS. There are several advantages of CED as compared to traditional delivery methods: 1) CED bypasses the BBB and can be used to infuse therapeutic agents with large or small molecular weights, and 2) it provides targeted delivery into the region of structure into which the catheter is placed. The ability to target delivery may serve to limit the potential for toxicity as systemic toxicity and widespread neurotoxicity can be avoided. Histological studies have shown that inflammation adjacent to the catheter tract and at the catheter tip is typically limited to within a $50 \mu \mathrm{m}$ radius. ${ }^{31}$ Furthermore, CED within the defined infusion measurements does not produce cerebral edema or measurable increases in intracranial pressure. ${ }^{32}$

For CED, one or more catheters are generally stereotactically implanted through a burr hole into or adjacent to either the enhancing portion of a tumor and/or the nonenhancing infiltrative surrounding tissue. A pressuredriven flow of the drug is achieved via an infusion pump, and the agent is directly infused into the target tissue at a predetermined concentration, rate, and duration. The volume of distribution achieved using CED into brain tumors differs markedly from the normal brain. This difference may be due to a number of factors including increased interstitial fluid pressure within and adjacent to brain tumors as compared to normal brain and an altered BBB. The increased interstitial fluid pressure observed in brain tumor creates a pressure gradient that can potentially drive infusate out of high-pressure areas within the tumor into relatively low-pressure areas in surrounding normal tissues. This pressure gradient and the marked heterogeneity of drug distribution within the tumor itself are potential limiting factors in drug delivery by this method. ${ }^{33}$ 


\section{Factors affecting drug distribution in CED}

Several key factors exist that affect the distribution of solutes delivered using CED. These include infusion rate, cannula size, infusion volume, interstitial fluid pressure, particle characteristics, and tumor tissue structure. The poroelastic medium of the brain allows for bulk fluid flow of both small and large molecular weight agents through the interstitium. Interstitial pathways allow for convective transport of agents with various molecular weights and sizes. The bulk flow of fluid within the brain is dispersed along several paths. Over longer time periods, drug diffusion, loss within the capillaries, and drug metabolism determine the distribution of drug. Other factors that influence drug distribution are related to catheter-induced tissue damage and reflux of solute adjacent to the catheter, "intrinsic" backflow of solute, and air bubbles - all of which can provide unpredictable and undesirable paths for fluid flow. The problem of backflow may be significant in cortical infusions where egress of the agent, along the cannula insertion track can occur, leading to subsequent widespread distribution of the agent within the subarachnoid space and thereby limiting drug delivery to the target tissues and increasing the potential for widespread neurotoxicity.

\section{Novel catheters for CED}

The size and shape of the delivery catheters have also contributed to some of the shortcomings of CED. Multiport catheters have been developed to improve infusate distribution, but data from gel models have shown that only the proximal ports deliver infusate, rendering the remaining ports useless for delivery. ${ }^{34}$ A hollow fiber catheter with multiple fenestrations to increase the surface area of brain tissue into which a drug can be infused was reported by $\mathrm{Oh}$ et al. ${ }^{35}$ Compared with standard, single-port, clinical catheters, the hollow fiber catheter appeared to have the advantage of increasing the distribution of drug therapy and reducing backflow in that report. Studies in agarose gels show that smaller cannulas perform better than larger ones by decreasing backflow and convecting fluid at a greater rate. More sophisticated catheters designed to provide more efficient distributions are currently under development and their use in clinical trials are underway. Real-time visualization of the CED process in vivo is also possible using a variety of imaging agents, including those that can be visualized with computed tomography or MRI. Use of these tracers will provide an added level of assurance of therapeutic drug distribution and allow alterations to the infusion plan if there is reflux or aberrant delivery.

\section{Therapeutic agents delivered by CED}

Viral vectors. The use of CED for delivering gene therapy to treat malignant gliomas and neurodegenerative disorders has been widely investigated in pre-clinical models and clinical trials. Hadaczek et al. ${ }^{36}$ examined the dis- tribution of AAV2-thymidine kinase and AAV2-aromatic L-amino acid decarboxylase (AAV2-AADC) in monkey brains after CED and demonstrated that this method is an efficient method for delivery of the AAV2 vector.

A modified infusion procedure using a novel infusion device designed for use in humans was evaluated for delivery of recombinant adeno-associated virus (AAV2). ${ }^{37}$ AAV2 encoding the human aromatic L-amino acid decarboxylase gene (AAV-hAADC-2) was infused in the putamen in Parkinson's disease patients. Aromatic L-amino acid decarboxylase immunohistochemistry demonstrated that vector was distributed throughout the putamen, with no significant difference in volume of immunostaining. Human clinical trials are currently in the planning stage.

\section{Paclitaxel}

The safety and efficacy of intratumoral CED of paclitaxel in patients harboring recurrent GBMs was examined in a phase I/II clinical study. ${ }^{38}$ Effective convection was determined by the progression of the hyperintense signal within the tumor on diffusion-weighted images, which corresponded to progressive tumor lysis. Of the 15 patients that received 20 cycles of intratumoral CED of paclitaxel, five complete responses and six partial responses were observed, giving a response rate of $73 \%$. CED of paclitaxel was associated with a high anti-tumor response rate, although it was also associated with a significant incidence of treatment-associated complications. ${ }^{38}$

\section{Topotecan}

Initial studies of chronic topotecan infusion (a topoisomerase I inhibitorz) into rats bearing C6 gliomas showed more than a five-fold increase in survival times without significant neurological toxicity. ${ }^{39}$ Based on these results, a phase I trial of topotecan delivery to patients with recurrent GBM was initiated, but outcomes have not yet been reported as of the time of this writing. CED of nanoparticle liposome containing topotecan has also been studied in vitro using rat models with experimental brain tumor. CED using liposomal topotecan resulted in extended tumor residence time as compared to free topotecan, as well as producing a significant survival benefit. ${ }^{40}$

\section{Targeted immunotoxins}

Another class of agents that have been investigated for use in CED is the so-called "targeted immunotoxins." Protein toxins produced by bacteria represent novel and potent cytotoxic agents, which may be coupled to specific carrier-ligands used for cellular targeting. The carrier-ligand provides tumor-selective properties by recognition of a cell-surface receptor on the tumor and promotes binding of the toxin-carrier complex prior to entry into the cell. These toxins tend to be far more potent than traditional chemotherapeutic agents and direct delivery into the brain likely represents the only means by which they can be administered safely. 


\section{Transferrin/diphtheria toxin}

One of the first conjugated toxins consisted of transferrin conjugated to the diphtheria toxin (TF-CRM107). ${ }^{41}$ This agent, renamed "TransMid," was evaluated in phase I, II and III studies. The phase I and II studies provided evidence of good tumor responses in patients with malignant brain tumors without significant neurotoxicity. ${ }^{42}$ Of the 15 patients with recurrent glioma, 9 patients responded to therapy with a greater than $50 \%$ decrease in contrast-enhancing tissue with MRI and a median survival at week 74 . A prospective, randomized phase III study of this toxin was opened to study its efficacy, delivered via CED into unresectable tumors, and compared to best medical therapy for treatment of recurrent GBM. Unfortunately, the study was halted before completion of its accrual due to an intermediate futility analysis that indicated a less than $20 \%$ chance of a positive outcome for the study.

\section{IL-4 and IL-13 targeted toxins}

Clinical studies of CED utilizing interleukin (IL)linked cytotoxins have also showed promising results in early stage clinical trials. ${ }^{43}$ One such targeted antineoplastic that is administered by CED is Cintredekin Besudotox (NeoPharm, Inc., Lake Forest IL). This is a recombinant protein consisting of a single molecule composed of two parts: 1) interleukin-13 (IL-13) which binds to IL-13Ralpha2 receptors on tumor cells, and 2) a truncated form of the Pseudomonas exotoxin (PE38QQR), a cytotoxin, which causes destruction of the tumor cell once the molecule is absorbed. IL13 receptors are present in substantial numbers on malignant glioma cells, but only a minimal amount on healthy neurons and astrocytes. $^{44,45}$

The safety and toxicity profile of IL13-PE38QQR was examined in three multicenter phase I trials with a total of 53 patients. ${ }^{46}$ Patients were treated with either 1) sequential intratumoral administration of the drug with tumor resection 3 to 21 days later and 24 to 48 hours after resection by intraparenchymal drug administration, or 2) a single intraparenchymal administration 24 to 72 hours after tumor resection. For intratumoral administration, one or two catheters were used, whereas one to three catheters were inserted for intraparenchymal use.

Intratumoral administration measurements of IL13PE38QQR evaluated infusion durations from 48 to 96 hours and total volume range of 19.2 to $51.8 \mathrm{~mL}$ at total infusion rates of 0.4 to $0.54 \mathrm{~mL} / \mathrm{h}$. IL13-PE38QQR concentrations ranged from 0.25 to $2 \mu \mathrm{m} / \mathrm{mL}$. The results of these studies indicated that the optimal conditions for infusion of IL13-PE38QQR was to use two to four catheters placed into the peritumoral area during a separate procedure after a gross total surgical resection of the enhancing tumor. The optimal safe dose was found to be $0.50 \mu \mathrm{g} / \mathrm{mL}$.
The Phase III Randomized Evaluation of ConvectionEnhanced Delivery of IL13-PE38QQR with Survival Endpoint trial, also known as the PRECISE study, randomized patients 2:1 to receive IL13-PE38QQR or Gliadel. IL13-PE38QQR was administered over 96 hours via two to four intraparenchymal catheters placed 2 to 7 days after tumor resection. Gliadel was placed immediately after tumor resection. The primary endpoint was overall survival from time of randomization. Median survival was comparable between the two groups and hence the study failed to meet its goal of improving survival of more than $50 \% .^{47,48}$ A phase I study of CED of IL13PE38QQR followed by radiation therapy with and without temozolomide in newly diagnosed malignant glioma revealed a well-tolerated therapy in these patients. ${ }^{49}$

\section{IL-4}

Phase I and II clinical trials have also been conducted with IL-4(38-37)-PE38KDEL (previously known as NBI-3001, and now called PRX321, and currently held by Protox Therapeutics, Vancouver, BC $)^{50,51}$ This agent is a chimeric protein composed of circularly permuted IL-4 and a truncated form of Pseudomonas exotoxin (PE). The safety and tolerability, and response of intratumoral infusion of IL-4(38-37)-PE-38KDEL into recurrent malignant high-grade gliomas has been examined. ${ }^{52}$ IL-4(38-37)-PE-38KDEL was infused intratumorally over a 4- to 8-day period into gliomas in nine patients using one to three stereotactically placed catheters. The infusion resulted in tumor necrosis in six of the nine patients, as evidenced by diminished gadolinium enhancement on MRI. The safety and toxicity of IL-4(38-37)PE-38KDEL was also examined in a multicenter, doseescalation phase I/II trial with intratumoral administration in 31 patients with recurrent malignant glioma, and it was suggested that compared to historical control there was an increase in overall median survival. ${ }^{53}$ Further development of this agent is planned.

\section{TP-38}

Human malignant gliomas and many malignant tumors that metastasize to the brain frequently overexpress the epidermal growth factor receptor (EGFR), usually via amplification of the EGFR gene on chromosome $7 \mathrm{p} .{ }^{54}$ EGFR has two natural ligands, epidermal growth factor and tumor growth factor-alpha, which may be used to specifically target cytotoxic agents to EGFR-expressing glioma cells. TP-38 is a recombinant chimeric-targeted toxin composed of the EGFR binding ligand, TGF-alpha, and a genetically engineered form of the Pseudomonas exotoxin, termed PE-38. Replacement of the native-binding domain with tumor growth factor-alpha (producing the toxin TP-38) selectively targets the toxin to cells expressing EGFR. Binding specificity was demonstrated by the ability of nonradiolabeled TP-38 to block binding of ${ }^{125}$ I-epidermal growth factor to an EGFR-expressing 
nonsmall cell carcinoma cell line, and TP-38 was capable of inhibiting protein synthesis in two EGFR-expressing lung carcinoma cell lines. ${ }^{55}$

The therapeutic efficacy of TP-38 has been demonstrated in an athymic murine intracranial tumor model after implantation of epidermoid carcinoma A431 cell line into the caudate nuclei. Control mice implanted with A431 cells mixed with phosphate buffered saline survived only 19 days, whereas 90 and $100 \%$ of mice survived when treated with TP-38 at $1.5 \mathrm{microg} / \mathrm{mL}$ and $5.0 \mathrm{microg} / \mathrm{mL}$, respectively. In 2001, a phase I/II clinical trial examined the toxicity and response to CED of TP-38, revealing an acceptable safety profile of TP-38. ${ }^{55}$ The overall median survival after TP-38 therapy was 23 weeks (range, 1.1 to 83.1 weeks). Median survival for patients with residual disease at the time of TP-38 therapy was 18.7 weeks, whereas those without radiographic evidence of residual disease demonstrated a median survival of 32.9 weeks. A subsequent phase III trial was planned, but it has not been opened at the time of this writing.

\section{TGF-beta}

Antisense Pharma (BioPark, Regensburg, Germany) has initiated several phase I/II studies with infusion of TGF-beta, AP 12009, an antisense oligonucleotide that specifically targets the mRNA encoding transforming growth factor-beta 2 (TGFbeta 2). ${ }^{56}$ Overexpression of TGF-beta2 is a hallmark of various malignant tumors, including pancreatic carcinoma, malignant glioma, metastasizing melanoma, and metastatic colorectal carcinoma. This is due to the pivotal role of TGF-beta2, as it regulates key mechanisms of tumor development, namely immunosuppression, metastasis, angiogenesis, and proliferation. The open-label dose-escalation studies in recurrent or refractory high-grade glioma patients showed a median survival time after recurrence exceeding the current literature data for chemotherapy. Currently, a phase I/II study in advanced pancreatic carcinoma, metastatic melanoma, and metastatic colorectal carcinoma and a phase IIb study in recurrent or refractory high-grade glioma are ongoing.

\section{CONCLUSION}

Malignant gliomas remain one of the deadliest forms of brain cancer in humans. Despite the advances in treatment with surgery, radiation, and chemotherapy, the prognosis is poor and the median survival is less than 2 years. New efficient and safe chemotherapeutics for the treatment of brain tumors have been developed over the years, and many are still under investigation. As exciting as many of these agents are in the laboratory, they face delivery hurdles imposed by the physical and biological properties of the normal brain, and the tumor infiltrated brain, which limit their clinical efficacy against malig- nant gliomas. Simultaneous development of novel drugdelivery techniques will be required to realize the full potential of these new anti-cancer agents.

BBBD and CED-mediated delivery of therapeutic agents remain promising strategies for treating malignant gliomas. Although initial clinical trials have failed to show survival benefit for new agents delivered via this approach, multiple earlier stage trials have addressed only a fraction of the myriad of technical and technological issues that surround these novel approaches. Development of CED has been limited by the fact that both new technologies and novel therapeutic agents are being developed simultaneously. New trials are being planned to investigate agents that can be co-infused with radiographic tracers, as well as to evaluate novel catheters that avoid problems with backflow and will potentially provide more reliable drug distribution.

\section{REFERENCES}

1. Central Brain Tumor Registry of the United States (CBTRUS): Statistical report: primary brain tumors in the United States, 20002004. Chicago, Central Brain Tumor Registry of the United States, 2005.

2. Central Nervous System Cancers Practice Guidelines in Oncology. Vol 2. Jenkintown, PA: National Comprehensive Cancer Network; 2005.

3. Nieder C, Grosu AL, Molls M. A comparison of treatment results for recurrent malignant gliomas. Cancer Treatment Rev 2000;26: 397-409.

4. Nicholas MK, Prados MD, Larson DA, Gutin PH. Malignant astrocytomas. In: Black P MCL, Loeffler JS, eds. Cancer of the nervous system. Cambridge: Blackwell Science, 1997:464-491.

5. Greenwood J. Experimental manipulation of the blood-brain and blood-retinal barriers. In: Bradbury MWB Ed, the physiology and pharmacology of the blood-brain barrier. Germany, SpringerVerlag, 1992:459-486.

6. Neuwelt EA. Mechanisms of disease: The blood-brain barrier. Neurosurgery 2004;54:131-142.

7. Rapoport SL, Robinson PJ. Tight-junctional modification as the basis of osmotic opening of the blood-brain barrier. Ann NY Acad Sci 1987;481:250-267.

8. Williams PC, Henner WD, Roman-Goldstein S, et al. Toxicity and efficacy of carboplatin and etoposide in conjunction with disruption of the blood-brain tumor barrier in the treatment of intracranial neoplasms. Neurosurgery 1995;37:17-28.

9. Neuwelt EA, Frenkel EP, Rapoport SI, et al. Effect of osmotic blood-brain barrier disruption on methotrexate pharmacokinetics in the dog. Neurosurgery 1980;7:36-43.

10. Dahlborg SA, Henner WD, Crossen JR, et al. Non-AIDS primary CNS lymphoma: The first example of a durable response in a primary brain tumor using enhanced chemotherapy delivery without cognitive loss and without radiotherapy. Cancer J Sci Am 1996;2:168-174.

11. Kraemer DF, Fortin D, Doolittle ND, et al. Association of total dose intensity of chemotherapy in primary CNS lymphoma (human non-AIDS) and survival. Neurosurgery 2001;48:10331041 .

12. McAllister LD, Doolittle ND, Guastadisegni PE, et al. Cognitive outcomes and long-term follow-up after enhanced chemotherapy delivery for primary central nervous system lymphomas. Neurosurgery 2000;46:51-61.

13. Neuwelt EA, Goldman DL, Dahlborg SA, et al. Primary CNS lymphoma treated with osmotic blood-brain barrier disruption: Prolonged survival and preservation of cognitive function. J Clin Oncol 1991;9:1580-1590.

14. Neuwelt EA, Barnett PA, Glasberg M, et al. Pharmacology and neurotoxicity of Cis-diamminedichloroplatinum, bleomycin, 5-flu- 
orouracil, and cyclophosphamide administration following osmotic blood-brain barrier modification. Cancer Res 1983;43:5278-5285.

15. Cloughesy TF, Black KL. Pharmacological blood-brain barrier modification for selective drug delivery. J Neurooncol 1995;26: 125-132.

16. Bartus RT, Elliott PJ, Dean RL, et al. Controlled modulation of BBB permeability using the bradykinin agonist, RMP-7. Exp Neurol 1996;142:14-28.

17. Ford J, Osborn C, Barton T, Bleehan NM. A phase I study of intravenous RMP-7 with carboplatin in patients with progression of malignant glioma. Eur J Cancer 1998;34:1807-1811.

18. Hynynen K, Clement GT, McDonald N, et al. 500-element ultrasound phased array system for noninvasive focal surgery of the brain: A preliminary rabbit study with ex vivo human skulls. Magn Reson Med 2004;52:100-107.

19. Hynynen K, McDannold N, Vykhodtseva N, et al. Noninvasive MR imaging-guided focal opening of the blood-brain barrier in rabbits. Radiology 2001;220:640-646.

20. Treat LH, McDannold N, Hynynen K. Transcranial MRI-guided focused ultrasound-induced blood-brain barrier opening in rats (2:998-1000). IEEE Ultrasonics Symposium, Montreal, Canada, August 24-27, 2004.

21. Clement GT, Hynynen K. A noninvasive method for focusing ultrasound through the human skull. Phys Med Biol 2000;47:12191236.

22. Brem H, Mahaley MS Jr, Vick NA, et al. Interstitial chemotherapy with drug polymer implants for the treatment of recurrent gliomas. J Neurosurg 1991;74:441-446.

23. Westphal M, Ram Z, Riddle V, Hilt D, Bortey E, and the Executive Committee of the Gliadel Study Group. Gliadel wafer in initial surgery for malignant glioma: long-term follow-up of a multicenter controlled trial. Acta Neurochir (Wien) 2006; 148(3):269-275.

24. Walter KA, Cahan MA, Gur A, et al. Interstitial Taxol delivered from a biodegradable polymer implant against experimental malignant glioma. Cancer Res 1994;54:2207-2212.

25. Watts MC, Lesniak MS, Burke M, et al. Controlled release of Adriamycin in the treatment of malignant glioma (poster). In: American Association of Neurological Surgeons Annual Meeting, Denver, CO, 1997.

26. Menei P, Venier MC, Gamelin E, et al. Local and sustained delivery of 5-fluorouracil from biodegradable microspheres for the radiosensitization of glioblastoma: a pilot study. Cancer 1999;86: 325-330.

27. Tajika Y, Muragaki Y, Hiyama H, et al. Local chemotherapy with slowly-releasing anticancer drug polymers for malignant brain tumors. J Control Fel 1994;32:1-8.

28. DeMeco F, Li KW, Tyler BM, et al. Local delivery of mitoxantrone for the treatment of malignant brain tumors in rats. J Neurosurg 2002;97:1173-1178.

29. Bobo RH, Laske DW, Akbasak A, et al. Convection-enhanced delivery of macromolecules in the brain. Proc Natl Acad Sci 1994; 15;91(6):2076-2080.

30. Cunningham J, Pivirotto P, Bringas I, et al. Biodistribution of adeno-associated virus type- 2 in nonhuman primates after convection-enhanced delivery to brain. Mol Ther 2008;16(7):12671275.

31. Lonser RR, Walbridge S, Garmestani K, et al. Successful and safe perfusion of the primate brainstem: in vivo magnetic resonance imaging of macromolecular distribution during infusion. J Neurosurg 2002;97(4):905-913.

32. Chen MY, Lonser RR, Morrison PF, et al. Variables affecting convection-enhanced delivery to the striatum: a systematic examination of rate of infusion, cannula size, infusate concentration, and tissue-cannula sealing time. J Neurosurg 1999;90(2): $315-320$.

33. Vogelbaum MA. Convection enhanced delivery for the treatment of malignant gliomas: symposium review. J Neurooncol 2005; 73(1):57-69.

34. Raghavan R, Brady ML, Rodriquez-Ponce MI, Hartlep A, Pedain C, Sampson JH. Convection-enhanced delivery of therapeutics for brain disease, and its optimization. Neurosurg Focus 2006;20(4): E12.
35. Oh S, Odland R, Wilson $\mathrm{S}$, et al. Improved distribution of small molecules and viral vectors in the murine brain using a hollow fiber catheter. J Neurosurg 2007;107:568-577.

36. Hadaczek P, Kohutnicka M, Krauze MT, et al. Convection-enhanced delivery of adeno-associated virus type 2 (AAV2) into the striatum and transport of AAV2 within monkey brain. Hum Gene Ther 2006;17(3):291-302.

37. Sanftner LM, Sommer JM, Suzuki BM, et al. AAV2-mediated gene delivery to monkey putamen: evaluation of an infusion device and delivery parameters. Exp Neurol 2005;194(2):476-483.

38. Lidar Z, Mardor Y, Jonas T, et al. Convection-enhanced delivery of paclitaxel for the treatment of recurrent malignant glioma: a phase I/II clinical study. J Neurosurg 2004;100(3):472-479.

39. Kaiser MG, Parsa AT, Fine RL, et al. Tissue distribution and antitumor activity of topotecan delivered by intracerebral clysis in a rat glioma model. Neurosurgery 2000;47(6):1391-1398.

40. Saito R, Krauze MT, Noble CO, et al. Convection-enhanced delivery of Ls-TPT enables an effective, continuous, low-dose chemotherapy against malignant glioma xenograft model. Neuro Oncol 2006;8(3):205-214.

41. Laske DW, Youle RJ, Oldfield EH. Tumor regression with regional distribution of the targeted toxin TF-CRM107 in patients with malignant brain tumors. Nat Med 1997;3:1362-1368.

42. Weaver M, Laske DW. Transferrin receptor ligand-targeted toxin conjugate (Tf-CRM107) for therapy of malignant gliomas. J Neurooncol 2002;65(1):3-13.

43. Kunwar S. Convection enhanced delivery of IL13-PE38QQR for treatment of recurrent malignant glioma: presentation of interim findings from ongoing phase 1 studies. Acta Neurochir 2003;88: 105-111.

44. Debinski W, Obiri N, Pastan I, et al. A novel chimeric protein composed of interleukin 13 and pseudomonas exotoxin is highly cytotoxic to human carcinoma cells expressing receptors for interleukin 13 and interleukin 4. J Biol Chem 1995;270:1677516780.

45. Debinski W, Obiri N, Powers S, et al. Human glioma cells overexpress receptors for interleukin 13 and are extremely sensitive to a novel chimeric protein composed of interleukin 13 and pseudomonas exotoxin. Clin Cancer Res 1995;1:1253-1258.

46. Kunwar S, Chang SM, Prados MD, et al. Safety of intraparenchymal convection-enhanced delivery of cintredekin besudotox in earlyphase studies. Neurosurg Focus 2006;20(4):E15.

47. Vogelbaum MA. Convection enhanced delivery for treating brain tumors and selected neurological disorders: symposium review. J Neurooncol 2007;83:97-109.

48. Kunwar S, Westphal M, Medhorn M, et al. Results from PRECISE: a randomized phase 3 study in patients with first recurrent glioblastoma multiforme (GBM) comparing cintredekin besudotox (CB) administered via convection-enhanced delivery (CED) with gliadel wafers $(\mathrm{GW})$. Abstract from the Twelfth Annual Meeting of the Society for Neuro-Oncology; October, 2007.

49. Vogelbaum MA, Sampson JH, Kunwar S, et al. Convection-enhanced delivery of cintredekin besudotox (interleukin-13PE38QQR) followed by radiation therapy with and without temozolomide in newly diagnosed malignant gliomas: phase 1 study of final safety results. Neurosurgery 2007;61:1031-1038.

50. Kawakami M, Kawakami K, Puri RK. Interleukin-4-pseudomonas exotoxin chimeric fusion protein for malignant glioma therapy. J Neurooncol 2003;5(1):15-25.

51. Weber FW, Floeth F, Asher A, et al. Local convection enhanced delivery of IL4-Pseudomonas exotoxin (NBI-3001) for treatment of patients with recurrent malignant glioma. Acta Neurochir Suppl 2003;88:93-103.

52. Rand RW, Kreitman RJ, Patronas N, et al. Intratumoral administration of recombinant circularly permuted interleukin-4-pseudomonas exotoxin in patients with high-grade glioma. Clin Cancer Res 2000;6(6):2157-2165.

53. Weber F, Asher A, Bucholz R, et al. Safety, toleratbility, and tumor response of IL4-Pseudomonas exotoxin (NBI-3001) in patients with recurrent malignant glioma. J Neurooncol 2003;64(1-2):125137.

54. Wong AJ, Bigner SH, Bigner DD, et al. Increased expression of the epidermal growth factor receptor gene in malignant gliomas is 
invariably associated with gene amplification. Proc Natl Acad Sci 1987;84(19):6899-6903.

55. Sampson JH, Akabani G, Archer GE, et al. Progress report of a Phase I study of the intracerebral microinfusion of a recombinant chimeric protein composed of transforming growth factor (TGF)alpha and a mutated form of the Pseudomonas exotoxin termed
PE-38 (TP-38) for the treatment of malignant brain tumors. J Neurooncol 2003;65(1):27-35.

56. Schlingensiepen KH, Fischer-Blass B, Schmaus, S, et al. Antisense therapeutics for tumor treatment: the TGF-beta 2 inhibitor AP 12009 in clinical development against malignant tumors. Recent Results Cancer Res 2008;177:137-150. 